\title{
Mechanisms and Predictions of Drug-Drug Interactions of the Hepatitis C Virus Three Direct-Acting Antiviral Regimen: Paritaprevir/Ritonavir, Ombitasvir, and Dasabuvir ${ }^{\text {ฐ }}$
}

\author{
Mohamad Shebley, Jinrong Liu, Olga Kavetskaia, Jens Sydor, Sonia M. de Morais, Volker Fischer, \\ Marjoleen J.M.A. Nijsen, and Daniel A.J. Bow
}

\begin{abstract}
Drug Metabolism, Pharmacokinetics, and Bioanalysis (M.S., J.L., O.K., J.S., S.M.d.M., V.F., M.J.M.A.N., D.A.J.B.) and Clinical Pharmacology and Pharmacometrics (M.S.), AbbVie Inc., North Chicago, Illinois
\end{abstract}

Received December 12, 2016; accepted May 2, 2017

\section{ABSTRACT}

To assess drug-drug interaction (DDI) potential for the three directacting antiviral (3D) regimen of ombitasvir, dasabuvir, and paritaprevir, in vitro studies profiled drug-metabolizing enzyme and transporter interactions. Using mechanistic static and dynamic models, DDI potential was predicted for CYP3A, CYP2C8, UDPglucuronosyltransferase (UGT) 1A1, organic anion-transporting polypeptide (OATP) 1B1/1B3, breast cancer resistance protein (BCRP), and P-glycoprotein (P-gp). Perpetrator static model DDI predictions for metabolizing enzymes were within 2-fold of the clinical observations, but additional physiologically based pharmacokinetic modeling was necessary to achieve the same for drug transporters. When perpetrator interactions were assessed, ritonavir was responsible for the strong increase in exposure of sensitive CYP3A substrates, whereas paritaprevir (an OATP1B1/1B3 inhibitor) greatly increased the exposure of sensitive OATP1B1/1B3 substrates. The 3D regimen drugs are UGT1A1 inhibitors and are predicted to moderately increase plasma exposure of sensitive UGT1A1 substrates. Paritaprevir, ritonavir, and dasabuvir are BCRP inhibitors. Victim DDI predictions were qualitatively in line with the clinical observations. Plasma exposures of the 3D regimen were reduced by strong CYP3A inducers (paritaprevir and ritonavir; major CYP3A substrates) but were not affected by strong CYP3A4 inhibitors, since ritonavir (a CYP3A inhibitor) is already present in the regimen. Strong CYP2C8 inhibitors increased plasma exposure of dasabuvir (a major CYP2C8 substrate), OATP1B1/1B3 inhibitors increased plasma exposure of paritaprevir (an OATP1B1/1B3 substrate), and P-gp or BCRP inhibitors (all compounds are substrates of $\mathrm{P}$-gp and/or BCRP) increased plasma exposure of the 3D regimen. Overall, the comprehensive mechanistic assessment of compound disposition along with mechanistic and PBPK approaches to predict victim and perpetrator DDI liability may enable better clinical management of nonstudied drug combinations with the 3D regimen.

\section{Introduction}

It is estimated that 170 million people worldwide are chronically infected with hepatitis C virus (HCV) (World Health Organization, 2011). Recently, orally available direct-acting antiviral (DAA) agents and compounds in clinical development have emerged to cure HCVinfected patients (Burger et al., 2013). Successful HCV therapies may require a combination of DAAs similar to HIV regimens; in some cases, the use of pharmacokinetic (PK) enhancers such as ritonavir may be needed. Approved HCV protease inhibitors (e.g., telaprevir, boseprevir, and simeprevir) and polymerase inhibitors (e.g., prodrug sofosbuvir) are substrates and inhibitors of drug-metabolizing enzymes and transporters, which are regularly involved in DDIs (Maasoumy et al., 2013; Khalilieh et al., 2015; Kirby et al., 2015). AbbVie's three direct-acting antiviral

This research was supported by AbbVie.

M.S. and D.A.J.B. contributed equally to this work.

https://doi.org/10.1124/dmd.116.074518.

$\$$ This article has supplemental material available at dmd.aspetjournals.org.
(3D) regimen is an all-oral interferon-free combination of the protease inhibitor paritaprevir (identified by AbbVie and Enanta) coformulated with ritonavir as a systemic PK enhancer and the Nonstructural protein 5A (NS5A) inhibitor ombitasvir, plus the non-nucleoside polymerase inhibitor dasabuvir with and without ribavirin (Feld et al., 2014). Frequently, patients with $\mathrm{HCV}$ take concomitant medications to treat conditions related to HCV or other comorbidities (Burger et al., 2013), leading to polypharmacy with the potential for complex DDIs, which in turn becomes an important consideration for physicians in choosing the most appropriate DAA regimen (Soriano et al., 2015). These DDIs mainly lead to systemic PK changes, which could affect the pharmacodynamic response and/or safety of concomitantly administered drugs (e.g., in the case of HIV-HCV coinfected and post-transplant patients) and, in some cases, may require dose adjustment or therapeutic monitoring of the affected medication(s).

Similar to HIV treatments, it is challenging to predict or extrapolate complex DDIs for a given combination in the absence of data from DDI clinical trials. In addition, it is not feasible to study all drug combinations in dedicated healthy volunteer trials to assess DDIs for every drug and

ABBREVIATIONS: 3D, three direct-acting antiviral; AUC, area under the curve; AUCR, area under the curve ratio; BCRP, breast cancer resistance protein; CAUCR, combination area under the curve ratio; DAA, direct-acting antiviral; DDI, drug-drug interaction; DMSO, dimethylsulfoxide; FDA, U.S. Food and Drug Administration; HCV, hepatitis C virus; HLM, human liver microsome; MATE, multidrug and toxin extrusion protein; MTX, $\left[{ }^{3} \mathrm{H}\right]$ methotrexate; OATP, organic anion-transporting polypeptide; OCT, organic cation transporter; P-gp, P-glycoprotein; P450, cytochrome P450; PBPK, physiologically based pharmacokinetic; PCR, real-time polymerase chain reaction; PK, pharmacokinetic; TDI, time-dependent inhibition; UGT, UDP-glucuronosyltransferase. 
mechanism and with all possible concomitant medications. Therefore, predictive nonclinical models are essential to aid our understanding of the complex enzyme and transporter interplay for each drug within the $3 \mathrm{D}$ regimen. To assess DDI potential against drug-metabolizing enzymes and drug transporters, the absorption, distribution, metabolism, and excretion properties for each drug in the 3D regimen (including a major circulating metabolite of dasabuvir, M1) were determined in the context of the 3D regimen clinical exposure. Predictions were used to not only inform clinical DDI trial design but to also offer a mechanistic explanation for observed clinical DDIs.

\section{Materials and Methods}

\section{Metabolism Studies}

Cytochrome P450 and Uridine-Diphosphate Glucuronosyltransferase Inhibition. Cytochrome P450 (P450) and UDP-glucuronosyltransferase (UGT) reversible inhibition was determined by measuring the extent of enzyme probe substrate conversion to the corresponding specific metabolite in human liver microsomes (HLMs). Known inhibitors were included as positive controls for each enzyme isoform. Test compounds were incubated at concentrations up to $30 \mu \mathrm{M}$ for all $\mathrm{P} 450$ isoforms (100 $\mu \mathrm{M}$ for CYP2B6) and up to $50 \mu \mathrm{M}$ for all UGTs. The probe substrate concentration for each P450 or UGT isoform was close to the previously reported $K_{\mathrm{m}}$ values, and assay incubations (pH 7.4) contained NADPH (1 mM) in phosphate buffer $(50 \mathrm{mM})$ for P450 isoforms or UDPGA $(5 \mathrm{mM})$ in Tris-HCL buffer $(50 \mathrm{mM})$ with magnesium chloride $(10 \mathrm{mM})$ for UGT isoforms. Specific assay conditions are included in the Supplemental Tables 1 and 2. Sample analysis of various enzyme incubations was performed by liquid chromatography-mass spectrometry using synthetic standards of metabolites with internal standards. For time-dependent inhibition (TDI) studies, the primary incubations consisted of $1 \mathrm{mg} / \mathrm{ml} \mathrm{HLMs}$ with 10 and $50 \mu \mathrm{M}$ test compound or positive controls (known mechanism-based inactivators for each P450 isoform) and $1 \mathrm{mM}$ NADPH in potassium phosphate buffer $(100 \mathrm{mM}), \mathrm{pH} 7.4$, incubated at $37^{\circ} \mathrm{C}$ for 0 and specified time (t1) that ranged from 10-30 minutes see Supplemental Table 3). Residual enzyme activity was determined using the P450-specific metabolite as a marker of activity. TDI potential was measured by calculating the difference in the extent of conversion of each $\mathrm{P} 450$ substrate to its corresponding metabolite ( 0 minutes to t1). Probe substrates were used in excess amount concentrations $>4$-fold of $K_{\mathrm{m}}$ ) for each P450 isoform. Specific TDI assay conditions are included in Supplemental Table 3.

P450 Induction. P450 induction was assessed in human hepatocytes from three donors (two females and one male). Cryopreserved hepatocytes were thawed $\left(37^{\circ} \mathrm{C}\right)$, centrifuged $(10$ minutes, $100 \mathrm{~g})$ in cryopreserved hepatocyte recovery medium (Life Technologies, Rockville, MD), and resuspended in Williams' E medium (without phenol red) supplemented with dexamethasone $(0.1 \mu \mathrm{M})$, insulin $(0.0001 \%)$, penicillin and streptomycin $(100 \mathrm{IU} / \mathrm{ml})$, and newborn calf serum $(5 \%)$. Hepatocyte viability was assessed by Trypan blue exclusion and cells were seeded in collagen-coated $96-$ well plates (approximately $0.8-0.9$ million cells $/ \mathrm{ml}$ ). Cells were incubated at $37^{\circ} \mathrm{C}\left(95 \%\right.$ air $\left./ 5 \% \mathrm{CO}_{2}\right)$ for 4 to 5 hours to allow hepatocytes to attach and were then overlaid with Geltrex (Life Technologies) $(0.35 \mathrm{mg} / \mathrm{ml})$. Cells were incubated overnight and then treated with test compounds $(0.1-10 \mu \mathrm{M})$ or positive controls (50 $\mu \mathrm{M}$ omeprazole, CYP1A2; $50 \mu \mathrm{M}$ phenytoin, CYP2B6; $10 \mu \mathrm{M}$ rifampin, CYP3A4) in serum-free incubation medium (Williams' $\mathrm{E}$ medium plus a hepatocyte maintenance supplement pack; Thermo Fisher Scientific, Waltham, MA) with $0.1 \%$ dimethylsulfoxide (DMSO) for 48 hours (medium changed daily). At the end of the incubation, cell viability was assessed according to the manufacturer's instructions for PrestoBlue cell viability reagent (Life Technologies) before RNA was isolated using the MagMAX Express 96 RNA Isolation System (Life Technologies). P450 mRNA expression for CYP1A2, CYP3A4, and CYP2B6 was assessed by reverse transcriptase polymerase chain reaction (PCR) (ViiA 7 Real-Time PCR System; Thermo Fisher Scientific) using the TaqMan EZ RT-PCR kit and standard probe/primer sets for each P450 isoform (Supplemental Table 4). Human 28S was used as an internal control to account for any variability in RNA levels. Induction was assessed by comparing the P450 mRNA fold-change between test compound- and vehicle control (0.1\% DMSO)-treated cells, the percent induction of the positive controls of P450 mRNA by the test articles compared with the vehicle control $(0.1 \%$ DMSO), and the percent induction of the positive controls.
P450 Phenotyping. Each compound (1-5 $\mu \mathrm{M}$ with a tritium radiolabel tracer) was incubated with a panel of P450 (1A2, 2B6, 2C8, 2C9, 2D6, 3A4, 3A5) Supersomes (BD Gentest, Woburn, MA) at $100 \mathrm{nmol} / \mathrm{ml}$ in phosphate buffer (100 mM, pH 7.4) in triplicate. Radiodetection coupled with high-performance liquid chromatography was used to monitor metabolite formation. Reactions were supplemented with NADPH $(1 \mathrm{mM})$, conducted at $37^{\circ} \mathrm{C}$ for 45 minutes, and terminated by the addition of organic solvent $[1: 1$ acetonitrile/methanol $(\mathrm{v} / \mathrm{v})]$. The samples were centrifuged (4000 rpm, 30 minutes) and the reaction supernatant was analyzed. Ritonavir was not tested in this study; therefore, previously published results are reported (Kumar et al., 1996). If more than one metabolizing enzyme was identified from the Supersome studies, follow-up incubations with HLMs in the presence of known inhibitors of specific P450 isoforms were conducted. Only dasabuvir was studied with specific inhibitors $(0.03-1 \mu \mathrm{M}$ ketoconazole, CYP3A; 0.5-45 $\mu \mathrm{M}$ quercetin, CYP2C8); incubations of dasabu$\operatorname{vir}(0.25 \mu \mathrm{M})$ with HLMs $(0.5 \mathrm{mg} / \mathrm{ml})$ were conducted at $37^{\circ} \mathrm{C}$ for 60 minutes. Reactions were terminated and analyzed as described above. The contribution of each enzyme was assessed qualitatively based on the detection of metabolites peaks from incubations. Contributions of each P450 were estimated based on observed turnover in the Supersome incubations for all compounds, except for dasabuvir, in which final $\mathrm{P} 450$ contributions were calculated based on the chemical inhibition results with selective CYP3A and CYP2C8 inhibitors.

\section{Transporter Studies}

\section{Uptake Transporters.}

Substrate Studies. Human embryonic kidney cells stably expressing organic anion-transporting polypeptide (OATP) 1B1 or 1B3 (obtained from Dietrich Keppler, German Cancer Research Center, Heidelberg, Germany), organic anion transporter (OAT) 1 or OAT3, organic cation transporter (OCT) 1 or OCT2, and multidrug and toxin extrusion protein (MATE) 1 or MATE2K (obtained from Kathleen M. Giacomini, Department of Biopharmaceutical Sciences, School of Pharmacy, University of California, San Francisco, CA) were used for substrate and/or inhibition evaluation for each of the compounds in the 3D regimen. OATP1B3 used in these studies has a mutation at amino acid 112 (serine to alanine). This mutated form was shown to exhibit the same substrate specificity as the native form of OATP1B3 (Fahrmayr et al., 2010).

Assays were performed as previously described (Kikuchi et al., 2013; Chiou et al., 2014) with incubation time points optimized for each of the individual transporters. Reference substrates para-aminohippuric acid, estrone sulfate, and 1-methyl-4-phenylpyridinium were used for OAT1, OAT3, and OCT2/MATE1/ MATE2K, respectively. Substrate uptake studies for each compound in the 3D regimen were conducted at multiple concentrations. If active uptake was observed, uptake kinetics $\left(K_{\mathrm{M}}\right.$ and $\left.V_{\max }\right)$ were determined by plotting the rate of initial uptake ( $V_{\mathrm{o}}$, in picomoles per minute per milligram of protein) against the compound concentrations (micromoles per liter) using the Michaelis-Menten equation. $K_{\mathrm{M}}$ and $\mathrm{V}_{\max }$ were determined by using nonlinear regression curve fitting in GraphPad Prism software (GraphPad Software Inc., La Jolla, CA).

Inhibition Studies. For each of the transporters, uptake inhibition was determined by incubations in the presence of increasing concentrations of each of the test compounds. $\mathrm{IC}_{50}$ values for inhibition (if calculated) were determined by using nonlinear regression curve fitting in GraphPad Prism.

\section{Efflux Transporters.}

Substrate Studies. In vitro efflux substrate assays were performed using Madin-Darby canine kidney II cells stably expressing P-glycoprotein (P-gp) or breast cancer resistance protein (BCRP). The cell lines were originally obtained from Piet Borst (Netherlands Cancer Institute, Amsterdam, Netherlands) and modified to knockout the expression of endogenous canine P-gp (Gartzke and Fricker, 2014). Cells were grown to confluence on Millicell 96-Cell Culture Plate polycarbonate filters, and substrate assays for each of the compounds in the $3 \mathrm{D}$ regimen were conducted as outlined in Gartzke and Fricker (2014). For ombitasvir efflux studies, assay conditions were modified to include $1 \%$ bovine serum albumin $(w / v)$ in the incubation medium to reduce nonspecific binding.

Inhibition Studies. P-gp and BCRP efflux inhibition was determined by incubation in the presence of increasing concentrations of each of the DAAs. Efflux ratios were obtained according to the following formula: Efflux ratio $=$ $\mathrm{P}_{\text {app }} \mathrm{B}$-to-A/ $\mathrm{P}_{\text {app }} \mathrm{A}$-to- $\mathrm{B}$, where $\mathrm{P}_{\text {app }}$ is the apparent permeability. $\mathrm{IC}_{50}$ values were determined in GraphPad Prism, with the following equation: $\mathrm{IC}_{50}=\log$ (inhibitor) versus response - variable slope. 
For membrane vesicle studies, assays were conducted according to the kit protocol (GenoMembrane, Kanagawa, Japan) with minor modifications. BCRPexpressing, Multidrug resistance-associated protein 2 (MRP2)-expressing, or control membrane vesicles were preincubated in transport buffer A at $37^{\circ} \mathrm{C}$ for 5 minutes and then rapidly mixed with an assay mixture containing $4 \mathrm{mM}$ ATP or AMP and the transporter probe substrate $100 \mu \mathrm{M}\left[{ }^{3} \mathrm{H}\right]-$ methotrexate (MTX; $10 \mu \mathrm{Ci} / \mathrm{ml}$ ) for BCRP or $50 \mu \mathrm{M}\left[{ }^{3} \mathrm{H}\right]$ estradiol $17-\beta$ glucuronide $(4 \mu \mathrm{Ci} / \mathrm{ml})$ for MRP2 in the presence or absence of a known inhibitor or test compound for 2 to 3 minutes. Transport was terminated by adding ice-cold wash buffer. The incubation mixture was filtered through a glass fiber filter plate and filters were washed with wash buffer. Radioactivity associated with each filter and incubation buffers was determined using a Micro- $\beta 2$ counter (PerkinElmer, Waltham, MA). ATP-dependent transport was calculated by subtracting the values obtained in the presence of AMP from those in the presence of ATP. Transporter-specific transport was calculated by subtracting the ATP-dependent MTX uptake in control vesicles from the ATP-dependent MTX uptake in the transporter-expressing vesicles. $\mathrm{IC}_{50}$ values were obtained by examining each compound's inhibitory effect on the ATPdependent transport of reference substrate assuming competitive inhibition. Fitting was performed by the nonlinear least-squares method using GraphPad Prism (version 5.04).

\section{Predictions of Cytochrome P450 and UGT Interactions}

Predictions of potential DDIs of the 3D regimen via P450 inhibition were made according to the 2012 U.S. Food and Drug Administration (FDA) draft guidance for industry on drug interaction studies (FDA, 2012) and following the recommendations in Fig. 4 of the FDA guidance using the mechanistic static model for reversible inhibition as follows:

$$
\begin{aligned}
& \mathrm{AUCR}=\left(\frac{1}{\left[A_{\mathrm{g}}\right] \times\left(1-F_{\mathrm{g}}\right)+F_{\mathrm{g}}}\right) \times\left(\frac{1}{\left[A_{\mathrm{h}}\right] \times f_{\mathrm{m}}+\left(1-f_{\mathrm{m}}\right)}\right) \\
& \text { Gut: } A_{g}=\frac{1}{1+\frac{[I]_{\mathrm{g}}}{K_{\mathrm{i}}}} \text {, where }[I]_{\mathrm{g}}=F_{\mathrm{a}} \times K_{\mathrm{a}} \times \text { Dose } / Q_{\mathrm{en}} \\
& \text { Liver: } A_{\mathrm{h}}=\frac{1}{1+\frac{[I]_{\mathrm{h}}}{K_{\mathrm{i}}}} \text {, where }[I]_{\mathrm{h}}=f_{\mathrm{u}, \mathrm{b}} \times\left([I]_{\mathrm{max}, \mathrm{b}}+F_{\mathrm{a}} \times K_{\mathrm{a}} \times \text { Dose } / Q_{\mathrm{h}}\right) \\
& \mathrm{cAUCR}=\left(\frac{1}{\left[A_{\mathrm{g}, \mathrm{comb}}\right] \times\left(1-F_{\mathrm{g}}\right)+F_{\mathrm{g}}}\right) \times\left(\frac{1}{\left[A_{\mathrm{h}, \mathrm{comb}}\right] \times f_{\mathrm{m}}+\left(1-f_{\mathrm{m}}\right)}\right) \\
& \text { Gut: } A_{\mathrm{g}, \text { comb }}=\frac{1}{1+\sum \frac{[I]_{\mathrm{g}, \text { drug } 1}}{K_{\mathrm{i}, \text { drug1 } 1}}+\frac{[I]_{\mathrm{g}, \text { drug } 2}}{K_{\mathrm{i} \text {, dug } 2}}+\ldots} \\
& \text { Liver: } A_{\mathrm{h}, \text { comb }}=\frac{1}{1+\sum \frac{\left[I_{\mathrm{h}, \text { dug1 } 1}\right.}{K_{\mathrm{i} \text {, drug } 1}}+\frac{[I]_{\mathrm{h} \text {, drug2 }}}{K_{\mathrm{i}, \text { dugg } 2}}+\ldots}
\end{aligned}
$$

The AUCR is the ratio of victim drug area under the curve (AUC) in the presence of the interacting drug relative to the control. cAUCR is a combination AUCR for a victim drug based on summing together ratios of the inhibitor in vivo concentration to in vitro $K_{\mathrm{i}}$ in the gut or liver of all drugs within the 3D regimen for a given P450 or UGT isoform. Subscripts " $h$ " and "g" denote the liver and gut, respectively; "comb" refers to parameters calculated for the combination; $F_{a}$ is the fraction absorbed after oral administration; $K_{\mathrm{a}}$ is the first order absorption rate constant in vivo; $Q_{\mathrm{en}}$ and $Q_{\mathrm{h}}$ are blood flow through enterocytes $(18 \mathrm{l} / \mathrm{h}$ per $70 \mathrm{~kg}$ ) and hepatic blood flow $(97 \mathrm{l} / \mathrm{h}$ per $70 \mathrm{~kg})$, respectively; $f_{\mathrm{m}}$ is the fraction of systemic clearance of the substrate mediated by the $\mathrm{P} 450$ enzyme that is subject to inhibition; $[I]_{\max , \mathrm{b}}$ is maximum systemic blood concentration; and $K_{\mathrm{i}}$ is the in vitro enzyme inhibition constant (FDA, 2012). For M1 only, prediction of UGT inhibition was also based on the ratio $\left(\mathrm{R}_{1}\right)$ of unbound $C_{\max }$ to in vitro enzyme $K_{\mathrm{i}}$.

Assumptions are as follows: $\mathrm{f}_{\mathrm{m}}$ of $\mathrm{P} 450$ probe substrates is assumed to be 0.99 (represents an upper estimate of the enzyme contribution; Brown et al., 2005), $\mathrm{F}_{\mathrm{g}}$ of midazolam as a CYP3A probe substrate was assumed to be 0.53 (Simcyp, version 14.1; Certara, Princeton, NJ), and $K_{\mathrm{a}}$ values used for each drug were previously reported (Mensing et al., 2016).

All drug parameters used in metabolism-based perpetrator DDI predictions are summarized in Table 2.

\section{Predictions of Transporter Interactions}

DDI predictions for efflux, hepatic uptake, and renal transporter inhibition were made using decision trees in the FDA (2012) draft guidance for industry on drug interaction studies.

For predictions of efflux transport inhibition, the following equations were used:

$$
\begin{aligned}
& {[I]_{1} / \mathrm{IC}_{50}\left(\text { or } K_{\mathrm{i}}\right) \geq 0.1} \\
& {[I]_{2} / \mathrm{IC}_{50}\left(\text { or } K_{\mathrm{i}}\right) \geq 10}
\end{aligned}
$$

where $[I]_{1}$ represents the mean steady-state total (free and bound) $C_{\max }$ after administration of the highest proposed clinical dose. $\left[I_{2}\right.$ is the dose of the inhibitor (in moles) $/ 250 \mathrm{ml}$ (if $\mathrm{IC}_{50}$ is in a molar unit) (FDA, 2012). For predictions of hepatic uptake inhibition, the following equations were used:

$$
\text { R-value }=1+\left(f_{\mathrm{u}} \times I_{\text {in,max }} / \mathrm{IC}_{50}\right)
$$

where R-value is the ratio of the victim drug AUC with inhibitor relative to the AUC without inhibitor, and $I_{\text {in,max }}$ is the estimated maximum inhibitor concentration at the inlet to the liver:

$$
I_{\text {in, } \max }=C_{\text {max }}+\left(K_{\mathrm{a}} \times \text { Dose } \times F_{\mathrm{a}} F_{\mathrm{g}} / Q_{\mathrm{h}}\right)
$$

where $C_{\max }$ is the maximum systemic plasma concentration of the inhibitor, dose is the inhibitor dose, $\mathrm{F}_{\mathrm{a}} \mathrm{F}_{\mathrm{g}}$ is the fraction of the dose of inhibitor that is absorbed, $K_{\mathrm{a}}$ is the absorption rate constant of the inhibitor and $Q_{\mathrm{h}}$ is the estimated hepatic blood flow (1500-1600 $\mathrm{ml} / \mathrm{min})$.

For renal transporters, predictions were based on $C_{\text {max, }} / \mathrm{IC}_{50}$ with a cutoff of 0.1 (FDA, 2012).

All drug parameters used in transporter-based perpetrator DDI predictions are summarized in Table 2.

\section{Physiologically Based Pharmacokinetic Modeling}

Pravastatin, rosuvastatin, and digoxin physiologically based pharmacokinetic (PBPK) substrate files were used directly from the Simcyp default "Substrate" library (version 14.1). These model files were verified against clinical data and published previously data (Varma et al., 2012; Neuhoff et al., 2013; Jamei et al., 2014). PBPK models for the $3 \mathrm{D}$ regimen as perpetrators were constructed using a combination of "top-down" and "bottom-up" approaches in Simcyp (version 14.1), using clinical PK data for paritaprevir, ritonavir, and dasabuvir and in vitro transporter inhibition data. Ombitasvir was not included in the PBPK model development process or the simulations, as transporter inhibition was not observed in vitro. Physical-chemical properties and observed oral clearance $(\mathrm{CL} / \mathrm{F})$ values from clinical PK studies for each drug were entered into Simcyp, and the volume of distribution $\left(V_{\mathrm{ss}}\right)$ was estimated using a minimal PBPK model (Rowland Yeo et al., 2010) with user input values and optimized parameter estimates (details in Supplemental Tables 5-7) to fit the PK profile of each drug. The disposition and elimination mechanisms of each drug were not assigned in the PBPK models because the models were intended to be used as perpetrators only. Absorption parameters $\mathrm{f}_{\mathrm{a}}$ and $k_{\mathrm{a}}$ were estimated using a top-down approach to match the $C_{\max }$ and $t_{\max }$ of each drug. Distribution parameters $V_{\mathrm{ss}}$, a single adjusting compartment, and intercompartmental clearance $(Q)$ were estimated using a top-down approach to match the observed shape of the PK curves for each drug. Because paritaprevir is a substrate for hepatic uptake transporters (Table 1), the liver-to-plasma ratio was assumed to be higher than unity. The paritaprevir final PBPK model was optimized by applying parameter estimation to the liver-toplasma partition coefficient $\left(K_{\mathrm{p}}\right)$ and the hepatic uptake factor of paritaprevir using clinical PK data (Supplemental Table 5). Validation of the 3D PBPK models was achieved by matching simulation results with observed clinical PK profiles of the $3 \mathrm{D}$ regimen. For DDI simulations with the $3 \mathrm{D}$ regimen, in vitro transporter $\mathrm{IC}_{50}$ values for each drug were used to predict the observed DDIs by the final PBPK models. For all DDI simulations, virtual trial designs (using a single population representative) in Simcyp were matched with clinical study designs. For pravastatin and rosuvastatin, $150 \mathrm{mg}$ paritaprevir daily with $100 \mathrm{mg}$ ritonavir daily and $400 \mathrm{mg}$ dasabuvir twice daily was simulated for 14 days with $10 \mathrm{mg}$ pravastatin daily or $5 \mathrm{mg}$ rosuvastatin daily under fed conditions. For digoxin, a 0.5 -mg single dose of digoxin was administered on day 15 after $150 \mathrm{mg}$ 
TABLE 1

In vitro enzyme and transporters victim data summary of paritaprevir, ritonavir, ombitasvir, dasabuvir, and M1

\begin{tabular}{|c|c|c|c|c|c|}
\hline $\begin{array}{l}\text { Enzyme or Transporter } \\
\text { (Endpoint or Unit) }\end{array}$ & Paritaprevir & Ritonavir & Ombitasvir & Dasabuvir & M1 \\
\hline CYP2C8 (\% contribution) & - & - & - & $\sim 60$ & $<10$ \\
\hline CYP3A4 (\% contribution ${ }^{a}$ ) & $\begin{array}{l}\sim 100,<50 \text { with } \\
\text { ritonavir }\end{array}$ & $\begin{array}{l}\sim 100,<50 \text { due to } \\
\text { autoinhibition }\end{array}$ & $\begin{array}{l}\sim 100,<10 \\
\text { with ritonavir }\end{array}$ & $\begin{array}{l}\sim 30,<10 \\
\text { with ritonavir }\end{array}$ & $\begin{array}{l}\sim 100,<10 \\
\text { with ritonavir }\end{array}$ \\
\hline P-gp/MDR-1 (net efflux ratio ${ }^{b}$ ) & 13 & 26 & 44 & 6 & 32 \\
\hline BCRP (net efflux ratio) & 5 & - & - & 2 & 2 \\
\hline OATP1B1, $K_{\mathrm{m}}(\mu \mathrm{M})^{c}$ & 0.2 & - & - & - & 1.3 \\
\hline OATP1B3, $K_{\mathrm{m}}(\mu \mathrm{M})$ & 0.1 & - & - & - & 1.7 \\
\hline OCT1 & - & - & - & - & Substrate \\
\hline
\end{tabular}

P450 contribution results are from incubations with individual recombinant human isoforms. Results for dasabuvir are based on chemical inhibition in human liver microsomes, and those for ritonavir are based on correlation analysis in human liver microsomes. Dashes indicate not a substrate based on in vitro studies. MDCKII, Madin-Darby canine kidney II.

${ }^{a} \mathrm{CYP} 3 \mathrm{~A} 4$ contribution is shown as individual drug in vitro results first, followed by the overall contribution within the 3D regimen where ritonavir is coadministered

${ }^{b}$ Efflux ratio from MDCKII cells overexpressing P-gp or BCRP (normalized for efflux in wild-type MDCKII cells).

${ }^{c}$ The Michaelis-Menton kinetics constant $\left(K_{\mathrm{m}}\right)$ was generated after initial active uptake screening studies (see the Materials and Methods).

paritaprevir daily with $100 \mathrm{mg}$ ritonavir daily and $400 \mathrm{mg}$ dasabuvir twice daily for 20 days under fed conditions. For comparison with the predicted DDI ratios, published clinical $C_{\max }$ and AUC geometric mean ratios of the victim drugs were used (Menon et al., 2015).

\section{Results}

\section{Metabolism Data}

Perpetrator Profile. In vitro inhibition data for P450s and UGTs by paritaprevir, ritonavir, ombitasvir, and dasabuvir and its major metabolite M1 are summarized in Supplemental Table 8. Ritonavir UGT inhibition was not studied; the literature value for UGT1A1 inhibition is reported in Supplemental Table 8 (Zhou et al., 2011). For reversible inhibition of P450s, paritaprevir and ombitasvir inhibited CYP2C8. Ritonavir inhibited CYP2B6, CYP2C8/9/19, CYP2D6, and CYP3A. Dasabuvir, but not M1, inhibited CYP2B6, CYP2C8/9/19, and CYP2D6 with relatively much weaker potency compared with ritonavir. All drugs in the 3D regimen inhibited UGT1A1. Paritaprevir, dasabuvir, and M1 inhibited UGT1A4; paritaprevir and dasabuvir inhibited UGT1A6; dasabuvir and M1 inhibited UGT1A9; and dasabuvir only inhibited UGT2B7.

For TDI of P450s, ritonavir is a potent inhibitor of CYP3A4 (20\%$77 \%$ preincubation effect at 0.1 and $1 \mu \mathrm{M}$, respectively), whereas paritaprevir and M1 exhibited minimal TDI on CYP3A4 (approximately $10 \%-15 \%$ preincubation effect at 10 and $50 \mu \mathrm{M}$, respectively). TDI parameters such as the inhibition constant $\left(K_{\mathrm{I}}\right)$ and the first order rate of inactivation constant $\left(k_{\text {inact }}\right)$ were not determined for paritaprevir and M1 due to the weak TDI observed in vitro, and they were not obtainable for ritonavir because of overlapping reversible inhibition potency with the TDI effect in vitro. Therefore, TDI-based predictions using static models were not attempted.

In human primary hepatocytes, ritonavir potently induced CYP3A4 mRNA ( $83 \%$ of rifampin response, at $10 \mu \mathrm{M}$ ), and paritaprevir showed weaker induction (30\% of rifampin response, at $10 \mu \mathrm{M}$ ) (Supplemental Table 9).

Victim Profile. Recombinant P450 phenotyping studies indicated that paritaprevir, ombitasvir, and M1 are predominantly metabolized by CYP3A4 with no metabolism observed by other P450s. There was some turnover of paritaprevir in CYP3A5 incubations; however, the contribution of CYP3A5 is considered negligible, since its intestinal and hepatic expression is relatively much lower compared with CYP3A4. Although ombitasvir and M1 appear predominately metabolized by CYP3A4 in vitro, ombitasvir is primarily metabolized by non-P450 amide hydrolysis pathways in vivo (little to negligible contribution by CYP3A4) and M1 is eliminated in vivo (Shen et al., 2016a) primarily unchanged via the hepatobiliary and urinary routes (minimal metabolism via $\mathrm{P} 450 \mathrm{~s}$ and direct glucuronidation). Dasabuvir was metabolized predominantly by CYP2C8 with partial metabolism by CYP3A (Table 1) based on results from the chemical inhibition study.

\section{Transporter Data}

Perpetrator Profile. In vitro inhibition data for efflux and uptake transporters are summarized in Supplemental Table 8. Paritaprevir, ritonavir, dasabuvir, and M1 inhibited P-gp and BCRP as well as the hepatic uptake transporters OATP1B1 and OATP1B3. Ritonavir inhibited OCT1. Renal transporters OAT1 and OAT3 were inhibited by paritaprevir and ritonavir. Ritonavir and M1 inhibited MATE1 and MATE2K, and paritaprevir inhibited MATE2K.

Victim Profile. All drugs in the 3D regimen and M1 are P-gp substrates, whereas only paritaprevir, dasabuvir, and M1 are BCRP substrates. Paritaprevir and M1 are substrates for the hepatic uptake transporters OATP1B1 and OATP1B3, and M1 is a substrate for OCT1 (Table 1). Because paritaprevir, ritonavir, ombitasvir, and dasabuvir have minimal renal elimination, in vitro renal transport substrate studies were not conducted.

\section{Metabolism-Mediated DDI Predictions}

For better understanding of the overall effects and predicted DDIs with the $3 \mathrm{D}$ regimen, individual and combined predictions were determined and explained in the context of a combination drug regimen. Predictions of the metabolism-based DDI potential of the 3D regimen were based on clinical doses, plasma exposures, and PK parameters (Table 2), and FDA-recommended basic and mechanistic static models (eq. 1) were used with modification to predict the combination effect (eq. 2) (FDA, 2012).

Inhibition Potential. Ritonavir had the highest predicted AUCR with CYP3A4 (AUCR of 38) (Table 3). In a clinical study with the 3D regimen and CYP3A-sensitive substrate tacrolimus, ritonavir caused a strong interaction (observed AUCR of 57) (Viekira Pak, 2014), consistent with the predicted AUCR of 28 and within 2-fold. The predicted CYP2C9 individual AUCR and cAUCR values for dasabuvir and ritonavir were below the FDA cutoff of 1.25 (Table 3), suggesting a low inhibition potential. Clinical interaction with warfarin as a CYP2C9 probe substrate resulted in an AUCR of 0.9 (Table 3) consistent with a predicted AUCR within 1.3-fold, which confirmed the absence of a clinically significant CYP2C9 interaction by the 3D regimen (Menon et al., 2015). The predicted CYP2C19 cAUCR was consistent with the clinical results of omeprazole (CYP2C19 substrate), in which clinical inhibition of CYP2C19 after coadministration of the 3D regimen was not 
TABLE 2

Parameters used in predictions of the 3D regimen as perpetrators of enzyme and transporter inhibition using basic and mechanistic static models

\begin{tabular}{|c|c|c|c|c|c|c|c|c|c|}
\hline Drug & Molecular Weight & Dose & $\mathrm{f}_{\mathrm{u}, \mathrm{p}}$ & $\mathrm{f}_{\mathrm{u}, \mathrm{b}}$ & $C_{\max , \mathrm{ss}}$ or []$_{1}$ & {$\left[I_{\mathrm{h}}\right.$} & {$\left[I_{2}\right]$} & $\mathrm{F}_{\mathrm{a}}$ or $\mathrm{F}_{\mathrm{a}} \mathrm{F}_{\mathrm{g}}$ & $K_{\mathrm{a}}$ \\
\hline & $\mathrm{g} / \mathrm{mol}$ & $m g$ & & \multicolumn{5}{|c|}{$n g / m l$} & $h^{-1}$ \\
\hline Paritaprevir & 765.89 & 150 & 0.020 & 0.029 & 1470 & 54.6 & 600 & 1 & 0.25 \\
\hline Ritonavir & 720.96 & 100 & 0.011 & 0.018 & 1600 & 32.5 & 100 & 1 & 0.17 \\
\hline Ombitasvir & 894.12 & 25 & 0.0003 & 0.0003 & 127 & 0.1 & 400 & 1 & 0.21 \\
\hline Dasabuvir & 493.58 & 250 & 0.004 & 0.006 & 1030 & 14.8 & 1000 & 1 & 0.58 \\
\hline M1 & 509.58 & - & 0.066 & 0.094 & 660 & - & - & - & - \\
\hline
\end{tabular}

Dashes indicate that parameters were not calculated for the metabolite, since it was not dosed orally. Generally, $\mathrm{f}_{\mathrm{u}, \mathrm{p}}$ was determined from in vitro studies, where the tested concentration of each drug is near its $C_{\mathrm{max}, \mathrm{ss}} . \mathrm{F}_{\mathrm{u}, \mathrm{b}}$ was calculated from $\mathrm{f}_{\mathrm{u}, \mathrm{p}}$ and the blood-to-plasma ratio. $\left[I_{\mathrm{h}}\right.$ is the hepatic inlet concentration as described in eq. $1 .\left[I_{2}\right.$ is oral dose amount of inhibitor $/ 250 \mathrm{ml}$ as described in eq. $2 . \mathrm{F}_{\mathrm{a}}$ and $\mathrm{F}_{\mathrm{g}}$ are fractions of the dose of inhibitor that was absorbed and escaped gut metabolism/extraction, respectively. $K_{\mathrm{a}}$ was estimated from clinical population PK analysis (Mensing et al., 2016).

observed (observed AUCR of 0.62) (Menon et al., 2015). In this clinical study, decreased exposure of omeprazole after coadministration with the 3D regimen can be explained by a net induction of CYP2C19 by ritonavir, which was reported previously (Yeh et al., 2006).

All compounds in the 3D regimen, including the M1 metabolite of dasabuvir, inhibit UGT1A1 in vitro with a range of potencies (Supplemental Table 8). Based on the same approach to predict reversible $\mathrm{P} 450$ inhibition DDIs using the mechanistic static model described above, only dasabuvir had a relatively higher predicted AUCR (1.06) but was still below the cutoff of 1.25, suggesting a remote potential for interaction with coadministered substrates of UGT1A1, whereas the cAUCR (1.20) predicted a weak interaction (Table 3). The predicted cAUCR of 1.20 was within 2-fold of a moderate and not clinically relevant observed AUCR of 2.4 with raltegravir (substrate of UGT1A1) (Menon et al., 2015).

Victim Potential. Based on in vitro studies, all drugs in the $3 \mathrm{D}$ regimen are CYP3A4 substrates with varying degrees of sensitivity and extent of contribution to their elimination (Table 1). The significant contribution of CYP3A4 to the elimination of paritaprevir was confirmed in clinical studies after coadministration with ritonavir in which paritaprevir exposure was markedly increased (Menon et al., 2015). Within the 3D regimen, only a moderate (observed AUCR of approximately 2) clinical interaction with ketoconazole (potent CYP3A4/P-gp inhibitor) was observed due to the presence of coformulated ritonavir (Menon et al., 2015).

Dasabuvir is predominantly metabolized by CYP2C8 (approximately $60 \%)$ and to a lesser extent by CYP3A4 (30\%) based on in vitro data from incubations with selective CYP2C8 and CYP3A inhibitors. A clinical study with dasabuvir and the potent CYP2C8 inhibitor gemfibrozil confirmed the significant contribution of CYP2C8 (observed AUCR of approximately 11), whereas the minor contribution of CYP3A4 (observed AUCR $<2$ ) was confirmed in a clinical study with ketoconazole coadministration (Menon et al., 2015).

Although CYP3A4 appeared to metabolize ombitasvir in vitro, the extent of metabolism is limited and the contribution of CYP3A4 to the overall elimination of ombitasvir is minimal due to the predominant amide hydrolysis pathway and the presence of coadministered ritonavir within the 3D regimen, consistent with the absence of interaction with ketoconazole (Menon et al., 2015).

Based on in vitro metabolism data of the predominant role of CYP3A, plasma exposures of all drugs within the $3 \mathrm{D}$ regimes were predicted to be significantly affected by strong CYP3A inducers. A significant decrease $(>30 \%)$ in paritaprevir, ritonavir, dasabuvir, and ombitasvir systemic exposures was observed after coadministration with the strong CYP3A inducer carbamazepine (Menon et al., 2015).

\section{Transporter-Mediated DDI Predictions}

Predictions of transporter-based DDIs of the 3D regimen were based on in vitro $\mathrm{IC}_{50}$ values, clinical doses, and plasma exposures (Table 2), as well as the FDA-recommended basic and mechanistic static models (eqs. 2-5) (FDA, 2012).

Inhibition Potential. Inhibition of intestinal efflux transporters (P-gp, BCRP) was predicted to be possible $\left(\left[I_{2}\right] / \mathrm{IC}_{50}\right.$ values $\left.>10\right)$ for paritaprevir, ritonavir, and dasabuvir but not likely for ombitasvir (Table 4). Inhibition of efflux transporters at other tissue sites (e.g., liver and kidney) was predicted for P-gp by ritonavir ([ $\left.I_{1}\right] / \mathrm{IC}_{50}$ value of 6.3 )

TABLE 3

Calculated model parameters and predicted AUCR values for the $3 \mathrm{D}$ regimen based on a mechanistic static model or basic model for reversible P450/UGT inhibition for individual components or the combined regimen

\begin{tabular}{|c|c|c|c|c|c|c|c|c|}
\hline \multirow[b]{2}{*}{ Enzyme } & \multirow{2}{*}{$\begin{array}{c}\text { Paritaprevir } \\
\text { Predicted } \\
\text { AUCR }\end{array}$} & \multirow{2}{*}{ 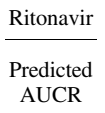 } & \multirow{2}{*}{ 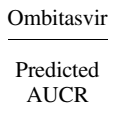 } & \multirow{2}{*}{$\begin{array}{c}\frac{\text { Dasabuvir }}{\text { Predicted }} \\
\text { AUCR }\end{array}$} & \multirow{2}{*}{$\frac{\mathrm{M} 1}{\text { Predicted } \mathrm{R}_{1}}$} & \multicolumn{3}{|c|}{ 3D Regimen } \\
\hline & & & & & & $\begin{array}{l}\text { Predicted } \\
\text { cAUCR }\end{array}$ & $\begin{array}{l}\text { Observed } \\
\text { cAUCR }\end{array}$ & $\begin{array}{l}\text { Predicted/ } \\
\text { Observed }\end{array}$ \\
\hline CYP2C9 & - & 1.17 & - & 1.01 & - & 1.17 & $0.9^{a}$ & 1.3 \\
\hline CYP2C19 & - & 1.02 & - & 1.00 & - & 1.02 & $0.6^{a}$ & 1.7 \\
\hline CYP3A4 & - & 28.2 & - & - & - & 28.2 & $57^{b}$ & 0.5 \\
\hline UGT1A1 & 1.04 & 1.05 & 1.00 & 1.06 & 1.03 & 1.20 & $2.4^{c}$ & 0.5 \\
\hline
\end{tabular}

Dashes indicate that parameters were not calculated because the $\mathrm{IC}_{50}$ could not be estimated due to no or low inhibition at the highest tested concentration in vitro. Predicted AUCR calculations for the reversible P450 inhibition using the mechanistic static model are described in eq. 1 . The predicted $\mathrm{R}_{1}$ calculation is from a modified basic model for predicting reversible $\mathrm{P} 450$ inhibition: $\mathrm{R}_{1}=1+[\mathrm{I}] / K_{\mathrm{i}}$, where [I] is the unbound $C_{\max }$ of the inhibitor and $K_{\mathrm{i}}\left(\right.$ or $\left.\mathrm{IC}_{50} / 2\right)$ is the in vitro inhibition constant. M1 is a metabolite of dasabuvir; thus, the $\mathrm{R}_{1}$ model was used for DDI predictions. cAUCR is the combination AUCR prediction (as described in Materials and Methods).

${ }^{a}$ Menon et al. (2015).

${ }^{b}$ Badri et al. (2015).

${ }^{c}$ Khatri et al. (2016). 
TABLE 4

Calculated model parameters and predictions of the 3D regimen as perpetrators of intestinal or systemic efflux transporters and hepatic uptake transporters

\begin{tabular}{|c|c|c|c|c|c|c|c|}
\hline \multirow{3}{*}{ Drug } & \multicolumn{4}{|c|}{ Intestinal/Hepatic/Renal } & \multicolumn{3}{|c|}{ Hepatic } \\
\hline & \multicolumn{2}{|c|}{ P-gp } & \multicolumn{2}{|c|}{ BCRP } & OATP1B1 & OATP1B3 & OCT1 \\
\hline & {$\left[\mathrm{I}_{2}\right] / / \mathrm{C}_{50}$} & {$\left[\mathrm{I}_{1}\right] / \mathrm{CC}_{50}$} & {$\left[\mathrm{I}_{2}\right] / / \mathrm{CC}_{50}$} & {$\left[\mathrm{I}_{1}\right] / / \mathrm{CC}_{50}$} & & R-Value & \\
\hline Paritaprevir & 20.6 & 0.05 & 1328 & 3.25 & 2.6 & 3.9 & - \\
\hline Ritonavir & 1585 & 6.34 & 23.1 & 0.09 & 1.05 & 1.05 & 1.01 \\
\hline Ombitasvir & - & - & - & - & - & - & - \\
\hline Dasabuvir & 121 & 0.12 & 130 & 0.13 & 1.02 & 1.00 & - \\
\hline M1 & - & 0.02 & - & 0.02 & 1.03 & 1.01 & - \\
\hline
\end{tabular}

[]$_{1}$ represents the mean steady-state total (free and bound) $C_{\max }$ after administration of the highest proposed clinical dose as described in eq. $3 .[]_{2}$ is oral dose of inhibitor $/ 250 \mathrm{ml}$ as described in eq. 4. Dashes indicate that parameters were not calculated because the $\mathrm{IC}_{50}$ could not be estimated due to no or low inhibition at the highest tested concentration in vitro. The R-value is the ratio of unbound inhibitor concentration at the inlet to the liver to in vitro $\mathrm{IC}_{50}$, as described in eq. 5 .

and for BCRP by paritaprevir $\left(\left[I_{1}\right] / \mathrm{IC}_{50}\right.$ value of 3.2$)$, whereas dasabuvir predictions were slightly above the cutoff point of 0.1 (Table 4). In clinical studies, a small increase (approximately 16\%) in systemic exposure of digoxin was observed with the $3 \mathrm{D}$ regimen (Menon et al., 2015). In efforts to further evaluate the transporterbased DDI predictions of the 3D regimen, PBPK modeling was employed. The PBPK models of paritaprevir, ritonavir, and dasabuvir were developed using a combined bottom-up (in vitro transporter $\mathrm{IC}_{50}$ values) and top-down (clinical PK parameters) approach. The developed models were calibrated using clinical PK data, in which absorption and distribution parameters were optimized for paritaprevir, ritonavir, and dasabuvir to fit each drug's clinical PK profile and parameters. The final models were in good agreement with the clinical data (Supplemental Figs. 1-3) and were simulated in combination during all DDI trial simulations. PBPK simulations of the $3 \mathrm{D}$ regimen at steady state with a single dose of digoxin (a P-gp substrate) on day 15 resulted in negligible changes in digoxin exposure. The predicted $C_{\max }$ ratio of 1.19 and the AUCR of 1.20 for digoxin after coadministration with the $3 \mathrm{D}$ regimen were within 1.25 -fold of the clinical results (Table 5). Effects of the 3D regimen on BCRP were simulated using rosuvastatin as a probe substrate, and the predicted $C_{\max }$ ratio of 3.4 and the AUCR of 2.4 were within 2-fold of the clinical results (Table 5).

For hepatic uptake transporters, R-values of OATP1B1 and OAT1B3 by paritaprevir (2.6 and 3.9, respectively; Table 4) suggested a moderate DDI but were quantitatively overpredictive of the clinical interaction with the OATP1B1/1B3 substrate pravastatin (observed AUCR of 1.82) and the mixed OATP1B1/1B3 and BCRP substrate rosuvastatin (observed AUC of 2.59) systemic exposures (Menon et al., 2015). PBPK simulations of the $3 \mathrm{D}$ regimen drugs as perpetrators with pravastatin and rosuvastatin significantly improved the predicted AUCRs and provided an advantage in predicting $C_{\max }$ ratios within the 2-fold acceptance criteria (Table 5) compared with the R-value static predictions. Although PBPK-based predictions were closer to the observed data compared with static models, there was a slight underprediction of the rosuvastatin $C_{\max }$ ratio compared with clinical observation (predicted versus observed, 3.4 versus 7.1); therefore, sensitivity analysis on intestinal BCRP $\mathrm{IC}_{50}$ values for paritaprevir, ritonavir, and dasabuvir was employed. The additional PBPK sensitivity analysis simulations revealed that only the paritaprevir $\mathrm{IC}_{50}$ was sensitive to intestinal BCRP interaction, where a 3-fold decrease in the paritaprevir $\mathrm{IC}_{50}$ resulted in an improvement in the predicted $C_{\max }$ ratio of 4.10 and the AUCR of 2.55. Further decrease (60- to 600-fold) in the paritaprevir BCRP $\mathrm{IC}_{50}$ value increased the $C_{\max }$ ratio up to a maximum of 5-fold, with a consistent underprediction of the observed value ( $C_{\max }$ ratio, 7.1-fold).

For renal transporters, unbound plasma concentrations of the $3 \mathrm{D}$ regimen were predicted to be insufficient to clinically inhibit OAT1, OAT3, OCT2, MATE1, or MATE2K. Consistently, clinical interaction with the OAT1 substrate tenofovir and OCT2 substrate metformin after coadministration with the 3D regimen was not observed (Menon et al., 2014, 2015; Polepally et al., 2016).

Victim Potential. As victims of transporters, paritaprevir, dasabuvir, and M1 are substrates for P-gp and BCRP, whereas ritonavir and ombitasvir are substrates for P-gp only (Table 1). Although ketoconazole's in vivo inhibitory contribution on efflux transporters versus CYP3A is difficult to discern, coadministration increased exposure of paritaprevir, ritonavir and dasabuvir, whereas ombitasvir was not affected (Menon et al., 2015).

Paritaprevir and M1 are substrates for OATP1B1 and OATP1B3 (Table 1) in vitro, and strong inhibitors were expected to increase paritaprevir or M1 systemic exposure. The systemic exposure of paritaprevir, but not M1, was sensitive to OATP inhibition after coadministration with the OATP inhibitors atazanavir and cyclosporine (Menon et al., 2015).

\section{Discussion}

In this study, in vitro metabolism and transporter interactions for paritaprevir, ritonavir, ombitasvir, dasabuvir, and M1 (a metabolite of dasabuvir) were characterized and extrapolated using static and dynamic DDI models. Clinical DDI studies were conducted during the development of the 3D regimen to evaluate interactions with P450s, UGTs, and drug transporters. The results from those DDI trials were in agreement with the predictions using in vitro data (Tables 1, 3, and 4).

\section{Perpetrator Profile}

All P450-mediated DDI predictions using static mechanistic models were within 2-fold of the observed data. Ritonavir was responsible for the majority of P450-mediated interactions as a strong inhibitor of CYP3A and a moderate inducer of CYP2C19 (Table 6). For UGTs, the cAUCR approach was able to predict a potential inhibitory interaction,

TABLE 5

PBPK model predictions of the 3D regimen as perpetrators of intestinal and hepatic efflux and hepatic uptake transporters compared with clinical results

\begin{tabular}{|c|c|c|c|c|c|c|}
\hline \multirow{2}{*}{$\begin{array}{c}\text { Victim Drug } \\
\text { (Exposure Ratios with Inhibitors) }\end{array}$} & \multicolumn{2}{|c|}{$\begin{array}{l}\text { Digoxin } \\
\text { (P-gp) }\end{array}$} & \multicolumn{2}{|c|}{$\begin{array}{c}\text { Rosuvastatin } \\
\text { (BCRP Plus OATP1B1/1B3) }\end{array}$} & \multicolumn{2}{|c|}{$\begin{array}{c}\text { Pravastatin } \\
\text { (OATP1B1/1B3) }\end{array}$} \\
\hline & Predicted & Observed $^{a}$ & Predicted & Observed $^{a}$ & Predicted & Observed $^{a}$ \\
\hline$C_{\max }$ ratio & 1.19 & 1.15 & $3.42,4.10^{b}$ & 7.13 & 2.0 & 1.37 \\
\hline AUC ratio & 1.20 & 1.16 & $2.41,2.55^{b}$ & 2.59 & 2.16 & 1.82 \\
\hline
\end{tabular}

${ }^{a}$ Menon et al. (2015)

${ }^{b}$ Results from sensitivity analysis simulations on paritaprevir and ritonavir in vitro $\mathrm{IC}_{50}$ values against intestinal BCRP. 
consistent with the observed DDI with a UGT1A1 substrate raltegravir (Menon et al., 2015) (Table 6). The proposed cAUCR is a simplistic approach for predicting effects of combination drugs on one mechanism; however, a similar approach for predicting metabolite-based DDIs (Templeton et al., 2016) was recently reported and may be justified for predicting drug combination DDIs. Overall, metabolism-based perpetrator predictions were in good quantitative agreement with clinical data when the 2-fold criterion was applied.

Paritaprevir, ritonavir, and dasabuvir were predicted to inhibit intestinal P-gp, whereas only ritonavir and dasabuvir were predicted to inhibit P-gp in the liver and kidney (Table 4). Only a minimal increase in the exposure (similar magnitude increase for both $C_{\max }$ and AUC) of digoxin was observed, suggesting that this interaction may have only occurred in the intestine. However, previous reports suggested the lack of sensitivity of digoxin to intestinal P-gp inhibition (Shi et al., 2011; Nader and Foster, 2014), and overprediction of the observed DDI by the static models may be explained in part by some of the assumptions in the calculations. For example, the static model assumes that the compounds are fully soluble in $250 \mathrm{ml}$ and, unlike the PBPK model, static modeling does not account for parameters such as permeability, transit time in the intestine, or compound solubility or dissolution. PBPK modeling significantly improved the DDI predictions of the $3 \mathrm{D}$ regimen with digoxin, where the predicted exposure ratios were within 1.25 -fold of the clinical results (Table 5). This improvement could be attributed to the fast absorption of digoxin relative to the $3 \mathrm{D}$ regimen $\left(t_{\max }\right.$ of approximately 1 hours versus 4 hours, respectively), as well as the low unbound concentrations of the $3 \mathrm{D}$ regimen.

For hepatic uptake transporters, only paritaprevir was predicted to inhibit OATPs (Table 4). A minimal increase in the exposure of pravastatin and a moderate increase in the exposure of the rosuvastatin were observed (Menon et al., 2015); however; static R-values overpredicted the inhibition potential of the $3 \mathrm{D}$ regimen with OATP and BCRP substrates. Refinement of the DDI prediction using PBPK modeling suggested a significant improvement in DDI predictions with pravastatin and rosuvastatin within 2-fold of the clinical results (Table 5). Unlike static models, PBPK modeling provides predictions for $C_{\max }$ ratios in addition to AUCs, which is an important advantage in the case of transporter interactions at the intestinal and hepatic levels, where $C_{\max }$ may be significantly affected. Sensitivity analysis using the developed PBPK models revealed that interaction with pravastatin is mainly due to significant inhibition of hepatic OATPs by paritaprevir, as well as interaction with rosuvastatin by inhibition of OATPs and BCRP by paritaprevir. This delineation of the main mechanism and perpetrator $\operatorname{drug}(\mathrm{s})$ that describes the observed interaction was possible because the Simcyp models of pravastatin and rosuvastatin explicitly described the contribution of each transporter mechanism, and the developed perpetrator models were also parameterized such that the in vitro $\mathrm{IC}_{50}$ or $K_{\mathrm{i}}$ for each transporter is entered separately and in each relevant organ (i.e., liver or gut). In fact, the predicted AUCRs for the $3 \mathrm{D}$ regimen with both statins were within 1.25-fold of the clinical observation, whereas predicted $C_{\max }$ ratios were within 2 -fold. Sensitivity analysis on paritaprevir BCRP $\mathrm{IC}_{50}$ values slightly improved the $C_{\max }$ ratio predictions, suggesting that the rosuvastatin model in Simcyp is potentially missing an intestinal and/or a hepatic transporter, which the 3D regimen inhibits. The PBPK approach improved accuracy of the DDI predictions to a satisfactory criterion within 1.25- to 2-fold of observed data, reflecting the robustness of the PBPK modeling approach; compared with the static basic and R-value models, a general conclusion regarding comparing the accuracy of these models cannot be drawn based on this small data set alone. However, the PBPK approach may be considered as a translational tool for predicting transporter-based DDIs with the $3 \mathrm{D}$ regimen as perpetrators based on the prediction results in Table 5, as well as for delineating the mechanisms of the complex interactions by the 3D regimen to allow for extrapolation to untested scenarios with concomitant medications. The presence of ritonavir within the 3D regimen may have complicated interpretation of the clinical DDI results; however, there are several clinical studies with ritonavir alone that may allow for delineating the observed DDI effects with the 3D regimen. For example, ritonavir alone did not alter the exposure of the OATP1B1 substrate pravastatin (Ieiri et al., 2013), whereas there was an approximately 2 -fold increase in pravastatin exposure with the $3 \mathrm{D}$ regimen, which was successfully predicted by PBPK simulations of the combined 3D regimen (Table 5) and was attributed to paritaprevir inhibition of OATP1B1.

Inhibition of renal transporters OAT1, OAT3, OCT2, MATE1, and MATE2K was also predicted to be clinically negligible. Consistent with these predictions, exposure of tenofovir (OAT1 substrate) and metformin (OCT2 substrate) did not change significantly after coadministration with the 3D regimen (Menon et al., 2014; Polepally et al., 2016).

TABLE 6

Summary of important mechanism-based DDI predictions and clinical observations of the 3D regimen

\begin{tabular}{|c|c|c|c|c|}
\hline \multirow{2}{*}{ Mechanism } & \multicolumn{2}{|c|}{ Perpetrator } & \multicolumn{2}{|c|}{ Victim } \\
\hline & DDI Predictions & Clinical Observations & DDI Predictions & Clinical Observations \\
\hline CYP3A & Ritonavir & Ritonavir & $\downarrow 3 \mathrm{D}$ regimen with strong inducers & $\downarrow 3 \mathrm{D}$ regimen with carbamazepine \\
\hline & $\uparrow$ Sensitive substrates & $\uparrow$ Paritaprevir and tacrolimus & 3D regimen with strong inhibitors & $3 \mathrm{D}$ regimen with ketoconazole \\
\hline CYP2C8 & None & - & $\uparrow$ Dasabuvir with strong inhibitors & $\uparrow$ Dasabuvir with gemfibrozil \\
\hline CYP2C19 & $\begin{array}{l}\text { Ritonavir and dasabuvir } \\
\text { Sensitive substrates }{ }^{a}\end{array}$ & $\begin{array}{l}\text { Ritonavir } \\
\downarrow \text { Omeprazole }^{b}\end{array}$ & None & - \\
\hline OATP1B1 and OATP1B3 & $\begin{array}{l}\text { Paritaprevir } \\
\uparrow \text { Sensitive substrates }\end{array}$ & $\begin{array}{l}\uparrow \text { Pravastatin } \\
\uparrow \text { Pravevir }\end{array}$ & $\uparrow$ Paritaprevir with strong inhibitors & $\uparrow$ Paritaprevir $^{b}$ with atazanavir \\
\hline BCRP & $\begin{array}{l}\text { Paritaprevir } \\
\uparrow \text { Sensitive substrates }\end{array}$ & $\begin{array}{l}\text { Paritaprevir } \\
\uparrow \text { Rosuvastatin }\end{array}$ & $\uparrow 3 \mathrm{D}$ regimen with strong inhibitors & $\uparrow 3 \mathrm{D}$ regimen $^{b}$ with cyclosporine \\
\hline UGT1A1 & $\begin{array}{l}\text { 3D regimen } \\
\uparrow \text { Sensitive substrates }\end{array}$ & $\begin{array}{l}\text { 3D regimen } \\
\uparrow \text { Raltegravir }^{b}\end{array}$ & None & - \\
\hline
\end{tabular}

Upward arrows, downward arrows, and horizontal arrows indicate an increase, decrease, or no change in systemic exposure, respectively. Dashes indicate that the mechanism was not tested in

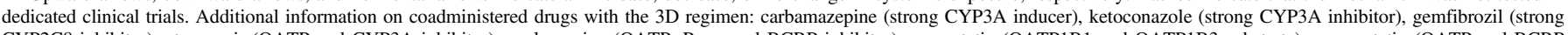

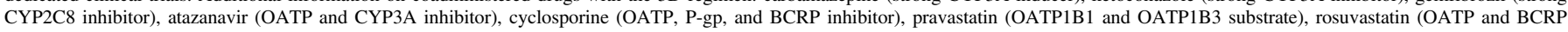
substrate), and raltegravir (UGT1A1 substrate).

${ }^{a} \mathrm{CYP} 2 \mathrm{C} 19$ induction was not considered in DDI predictions, due to unavailability of model parameters $\mathrm{EC}_{50}$ and $E_{\max }$ for ritonavir.

${ }^{b}$ The change in exposure is not clinically relevant (Badri et al., 2015; Khatri et al., 2016). 


\section{Victim Profile}

Coadministration of strong CYP2C8 inhibitors or inducers with dasabuvir may cause significant changes in dasabuvir exposure. Strong CYP3A inducers may cause a significant decrease in systemic exposure of all drugs within the 3D regimen, whereas strong CYP3A4 inhibitors may cause a modest increase in paritaprevir and ritonavir systemic exposures and minimal to no change to dasabuvir and ombitasvir exposures. The minimal inhibition of CYP3A is due to the presence of coadministered ritonavir and compensating non-P450-mediated amide hydrolysis of ombitasvir (Shen et al., 2016b). It should be noted that in the presence of ritonavir within the 3D regimen where CYP3A metabolism of paritaprevir is nearly shut down, the high distribution of paritaprevir to the liver is mainly driven by hepatic uptake transporters (OATP1B1/1B3). Thus, P450 inhibitors and probable inducers may only affect paritaprevir's systemic exposure but not liver exposure when used in combination with ritonavir, which is the target site of action.

Paritaprevir and M1, but not the other 3D components, are substrates for OATP1B1 and OATP1B3, whereas M1 is also a substrate of OCT1 (Table 1). These data are consistent with the observed increase in paritaprevir systemic exposure after coadministration with a mixed
A

Intestine

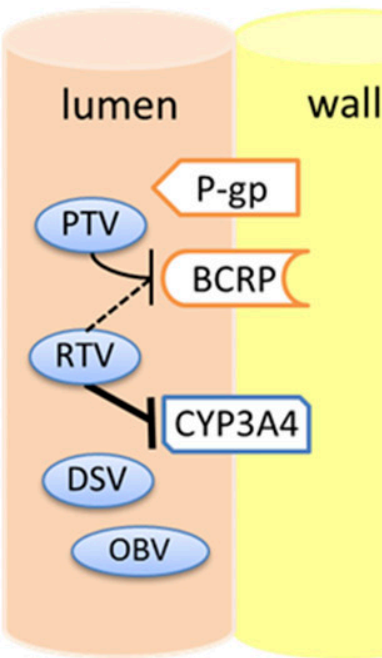

Blood

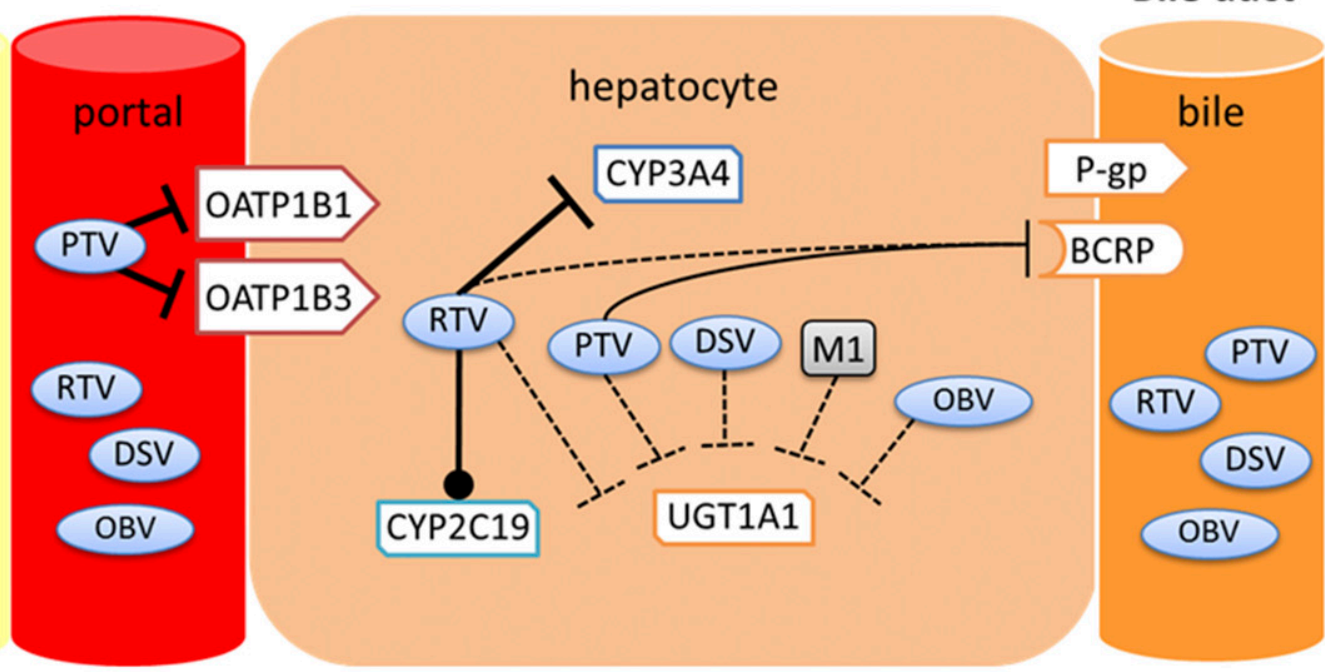

B

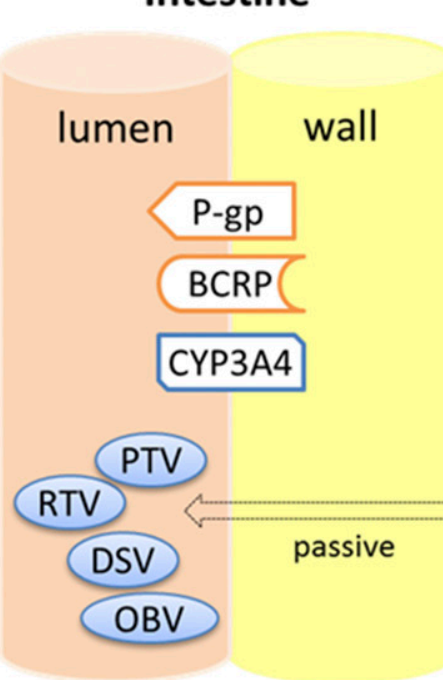

Blood

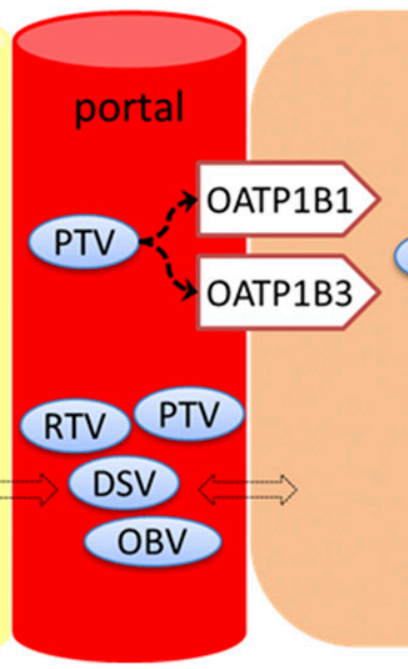

Bile duct

Liver

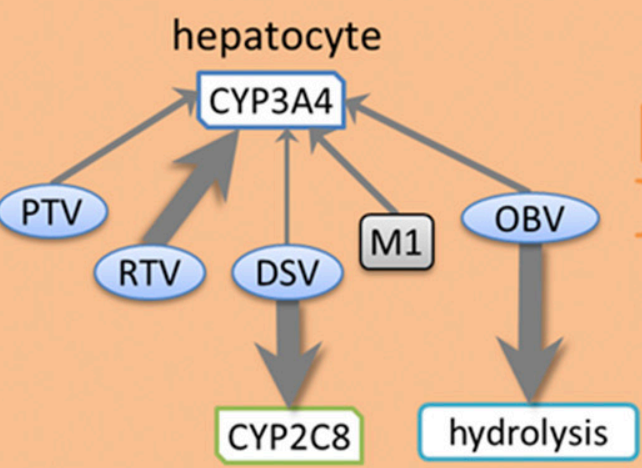

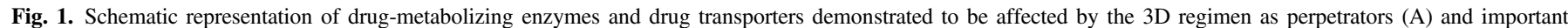
pathways involved in the disposition and elimination of the 3D regimen as victims (B). (A) Solid lines with a perpendicular endpoint represent strong inhibition of an enzyme/transporter, the solid line with a circular endpoint represents induction of an enzyme/transporter, and dashed lines represent weak inhibition of an enzyme or transporter. Ritonavir is responsible for intestinal and hepatic CYP3A4 net inhibition and DDIs with coadministered substrates, as well as moderate induction of CYP2C19. Paritaprevir is responsible for inhibition of OATP1B1, OATP1B3, and BCRP and resulting DDIs with coadministered substrates. The 3D regimen inhibits UGT1A1 and results in weak or moderate but not clinically relevant DDIs with coadministered substrates (e.g., raltegravir). (B) The intensity of the arrows represents the degree of pathway contribution to disposition and elimination of the 3D regimen, in which thick arrows represent a high contribution and vice versa. For simplicity, arrows are only shown in a hepatocyte in this schematic. Dashed arrows indicate the qualitative contribution of a corresponding mechanism. For CYP3A4 metabolism of drugs within the 3D regimen, coadministered ritonavir mostly diminishes the contribution of this pathway (see the Results and Discussion for more detail). DSV, dasabuvir; OBV, ombitasvir; PTV, paritaprevir; RTV, ritonavir. 
OATP/CYP3A inhibitor atazanavir (Khatri et al., 2016) (Table 6). M1 systemic exposure was not affected by atazanavir, suggesting that it is a product of dasabuvir metabolism in the liver rather than the intestine, such that M1 is likely not available in portal blood at the liver inlet where inhibition of hepatic uptake transporters would be greatest. Alternatively, M1 may have other routes of elimination, such as CYP3A hepatic metabolism (Table 1), and active hepatic uptake by OATPs may not be a rate-limiting step for its disposition and elimination.

In addition, the contribution of multiple transporters to paritaprevir disposition (Table 1) resulted in a modest increase in the systemic exposure of paritaprevir but not the other 3D components after coadministration with the mixed OATP, P-gp, BCRP, and CYP3A4 inhibitor cyclosporine (Menon et al., 2015).

The minimal to modest increase in the systemic exposure of the $3 \mathrm{D}$ regimen after coadministration of a mixed CYP3A/P-gp inhibitor ketoconazole suggests a possible contribution of $\mathrm{P}$-gp to individual drugs within the $3 \mathrm{D}$ regimen. The contribution of BCRP was indirectly assessed clinically, where only the exposure of paritaprevir increased after coadministration with a single 30-mg dose of cyclosporine (OATP, P-gp, BCRP, and CYP3A4 inhibitor) (Menon et al., 2014) (Table 6), although this mixed interaction could be attributed to OATP inhibition alone. Thus, for transporter-mediated victim DDIs, a qualitative assessment of the contribution of each transporter may be possible based on nonclinical and clinical studies with the 3D regimen, and a more mechanistic approach (e.g., PBPK) may be needed due to the complex interplay between metabolizing enzymes and transporters (Fig. 1B).

Overall, for metabolism-mediated DDIs, ritonavir predominates as a metabolism perpetrator (Fig. 1A) leading to a net inhibition of CYP3A at the 100-mg boosting dose. For transporter-mediated DDIs, paritaprevir and possibly ritonavir predominate as perpetrators leading to a net inhibition of OATPs, BCRP, and a relatively mild or negligible inhibition of P-gp (Fig. 1A). Drugs that are sensitive substrates of CYP3A, OATPs, and BCRP and have a narrow therapeutic window are expected to have DDIs upon coadministration with the 3D regimen (Table 6). Victim profiles of the $3 \mathrm{D}$ regimen are depicted in Fig. $1 \mathrm{~B}$, and semiquantitative contributions of drug-metabolizing enzymes are shown, whereas involvement of transporters is described qualitatively. Plasma exposures of the $3 \mathrm{D}$ regimen in combination with ritonavir were sensitive to strong CYP3A inducers but were not significantly affected by strong CYP3A inhibitors, and only the plasma exposure of dasabuvir was sensitive to strong inhibitors of CYP2C8. Inhibitors of P-gp or BCRP increased plasma exposures of the $3 \mathrm{D}$ regimen, whereas plasma exposure of only paritaprevir was increased by OATP1B1/1B3 inhibitors (Table 6). It may be possible, in the future, to achieve a more quantitative extrapolation of the $3 \mathrm{D}$ regimen victim interactions with perpetrator drugs if PBPK models are constructed for each of the compounds in the 3D regimen.

In conclusion, this comprehensive metabolism and transporter assessment of the $3 \mathrm{D}$ regimen, as well as the model-based approach to predicting DDIs, may enable better clinical management of nonstudied drug combinations with the $3 \mathrm{D}$ regimen.

\section{Acknowledgments}

The authors thank all members of the Drug Metabolism, Pharmacokinetics, and Bioanalysis Department who contributed to studies and analyses used in this article.

\section{Authorship Contributions}

Participated in research design: Shebley, Liu, Bow.

Conducted experiments: Shebley, Liu, Bow.

Performed data analysis: Shebley, Liu, Bow.

Wrote or contributed to the writing of the manuscript: Shebley, Liu, Kavetskaia, Sydor, de Morais, Fischer, Nijsen, Bow.

\section{References}

Badri P, Dutta S, Coakley E, Cohen D, Ding B, Podsadecki T, Bernstein B, Awni W, and Menon R (2015) Pharmacokinetics and dose recommendations for cyclosporine and tacrolimus when coadministered with ABT-450, ombitasvir, and dasabuvir. Am J Transplant 15:1313-1322.

Brown HS, Ito K, Galetin A, and Houston JB (2005) Prediction of in vivo drug-drug interaction from in vitro data: impact of incorporating parallel pathways of drug elimination and inhibitor absorption rate constant. Br J Clin Pharmacol 60:508-518.

Burger D, Back D, Buggisch P, Buti M, Craxí A, Foster G, Klinker H, Larrey D, Nikitin I, Pol S, et al. (2013) Clinical management of drug-drug interactions in HCV therapy: challenges and solutions. J Hepatol 58:792-800.

Chiou WJ, de Morais SM, Kikuchi R, Voorman RL, Li X, and Bow DA (2014) In vitro OATP1B1 and OATP1B3 inhibition is associated with observations of benign clinical unconjugated hyperbilirubinemia. Xenobiotica 44:276-282.

Fahrmayr C, Fromm MF, and König J (2010) Hepatic OATP and OCT uptake transporters: their role for drug-drug interactions and pharmacogenetic aspects. Drug Metab Rev 42:380-401.

FDA (2012) Drug Interaction Studies - Study Design, Data Analysis, Implications for Dosing, and Labeling Recommendations, U.S. Food and Drug Administration, Rockville, MD.

Feld JJ, Kowdley KV, Coakley E, Sigal S, Nelson DR, Crawford D, Weiland O, Aguilar H, Xiong J, Pilot-Matias T, et al. (2014) Treatment of HCV with ABT-450/r-ombitasvir and dasabuvir with ribavirin. $N$ Engl J Med 370:1594-1603.

Gartzke D and Fricker G (2014) Establishment of optimized MDCK cell lines for reliable efflux transport studies. J Pharm Sci 103:1298-1304.

Ieiri I, Tsunemitsu S, Maeda K, Ando Y, Izumi N, Kimura M, Yamane N, Okuzono T, Morishita M, Kotani N, et al. (2013) Mechanisms of pharmacokinetic enhancement between ritonavir and saquinavir; micro/small dosing tests using midazolam (CYP3A4), fexofenadine (p-glycoprotein), and pravastatin (OATP1B1) as probe drugs. J Clin Pharmacol 53:654-661.

Jamei M, Bajot F, Neuhoff S, Barter Z, Yang J, Rostami-Hodjegan A, and Rowland-Yeo K (2014) A mechanistic framework for in vitro-in vivo extrapolation of liver membrane transporters: prediction of drug-drug interaction between rosuvastatin and cyclosporine. Clin Pharmacokinet 53:73-87.

Khalilieh S, Feng HP, Hulskotte EG, Wenning LA, and Butterton JR (2015) Clinical pharmacology profile of boceprevir, a hepatitis C virus NS3 protease inhibitor: focus on drug-drug interactions. Clin Pharmacokinet 54:599-614.

Khatri A, Dutta S, Wang H, Podsadecki T, Trinh R, Awni W, and Menon R (2016) Evaluation of drug-drug interactions between hepatitis $\mathrm{C}$ antiviral agents ombitasvir, paritaprevir/ritonavir, and dasabuvir and HIV-1 protease inhibitors. Clin Infect Dis 62:972-979.

Kikuchi R, Lao Y, Bow DA, Chiou WJ, Andracki ME, Carr RA, Voorman RL, and De Morais SM (2013) Prediction of clinical drug-drug interactions of veliparib (ABT-888) with human renal transporters (OAT1, OAT3, OCT2, MATE1, and MATE2K). J Pharm Sci 102 4426-4432.

Kirby BJ, Symonds WT, Kearney BP, and Mathias AA (2015) Pharmacokinetic, pharmacodynamic, and drug-interaction profile of the hepatitis C virus NS5B polymerase inhibitor sofosbuvir. Clin Pharmacokinet 54:677-690.

Kumar GN, Rodrigues AD, Buko AM, and Denissen JF (1996) Cytochrome P450-mediated metabolism of the HIV-1 protease inhibitor ritonavir (ABT-538) in human liver microsomes. $J$ Pharmacol Exp Ther 277:423-431.

Maasoumy B, Port K, Calle Serrano B, Markova AA, Sollik L, Manns MP, Cornberg M, and Wedemeyer $\mathrm{H}$ (2013) The clinical significance of drug-drug interactions in the era of directacting anti-viral agents against chronic hepatitis C. Aliment Pharmacol Ther 38:1365-1372.

Menon R, Badri P, Khatri A, Wang T, Bow DA, Polepally A, Podsadecki T, Awni WM, and Dutta S (2014) ABT-450/ritonavir +ombitasvir + dasabuvir: drug interactions mediated by transporters in 15th International Workshop on Clinical Pharmacology of HIV and Hepatitis Therapy; 2014 May 19-21; Washington, DC.

Menon RM, Badri PS, Wang T, Polepally AR, Zha J, Khatri A, Wang H, Hu B, Coakley EP, Podsadecki TJ, et al. (2015) Drug-drug interaction profile of the all-oral anti-hepatitis C virus regimen of paritaprevir/ritonavir, ombitasvir, and dasabuvir. J Hepatol 63:20-29.

Mensing S, Polepally AR, König D, Khatri A, Liu W, Podsadecki TJ, Awni WM, Menon RM, and Dutta $S$ (2016) Population pharmacokinetics of paritaprevir, ombitasvir, dasabuvir, ritonavir, and ribavirin in patients with hepatitis $\mathrm{C}$ virus genotype 1 infection: combined analysis from 9 phase $1 \mathrm{~b} / 2$ studies. AAPS J 18:270-280.

Nader AM and Foster DR (2014) Suitability of digoxin as a P-glycoprotein probe: implications of other transporters on sensitivity and specificity. J Clin Pharmacol 54:3-13.

Neuhoff S, Yeo KR, Barter Z, Jamei M, Turner DB, and Rostami-Hodjegan A (2013) Application of permeability-limited physiologically-based pharmacokinetic models: part II - prediction of P-glycoprotein mediated drug-drug interactions with digoxin. J Pharm Sci 102:3161-3173.

Polepally AR, King JR, Ding B, Shuster DL, Dumas EO, Khatri A, Chiu YL, Podsadecki TJ, and Menon RM (2016) Drug-drug interactions between the anti-hepatitis C virus 3D regimen of ombitasvir, paritaprevir/ritonavir, and dasabuvir and eight commonly used medications in healthy volunteers. Clin Pharmacokinet 55:1003-1014.

Rowland Yeo K, Jamei M, Yang J, Tucker GT, and Rostami-Hodjegan A (2010) Physiologically based mechanistic modelling to predict complex drug-drug interactions involving simultaneous competitive and time-dependent enzyme inhibition by parent compound and its metabolite in both liver and gut - the effect of diltiazem on the time-course of exposure to triazolam. Eur J Pharm Sci 39:298-309.

Shen J, Serby M, Reed A, Lee AJ, Menon R, Zhang X, Marsh K, Wan X, Kavetskaia O, and Fischer V (2016a) Metabolism and disposition of hepatitis C polymerase inhibitor dasabuvi in humans. Drug Metab Dispos 44:1139-1147.

Shen J, Serby M, Surber B, Lee AJ, Ma J, Badri P, Menon R, Kavetskaia O, de Morais SM, Sydo $\mathrm{J}$, et al. (2016b) Metabolism and disposition of pan-genotypic inhibitor of hepatitis C virus NS5A ombitasvir in humans. Drug Metab Dispos 44:1148-1157.

Shi JG, Zhang Y, and Yeleswaram S (2011) The relevance of assessment of intestinal P-gp inhibition using digoxin as an in vivo probe substrate. Nat Rev Drug Discov 10:75-, author reply

Soriano V, Labarga P, Barreiro P, Fernandez-Montero JV, de Mendoza C, Esposito I, BenítezGutiérrez L, and Peña JM (2015) Drug interactions with new hepatitis C oral drugs. Expert Opin Drug Metab Toxicol 11:333-341.

Templeton IE, Chen Y, Mao J, Lin J, Yu H, Peters S, Shebley M, and Varma MV (2016) CPT Pharmacometrics. Syst Pharmacol 5(10):505-515. 
Varma MV, Lai Y, Feng B, Litchfield J, Goosen TC, and Bergman A (2012) Physiologically based modeling of pravastatin transporter-mediated hepatobiliary disposition and drug-drug interactions. Pharm Res 29:2860-2873.

Viekira Pak (2014) U.S. prescribing information. AbbVie Inc., North Chicago, IL.

World Health Organization (2011) Hepatitis C. Wkly Epidemiol Rec 86:445-447.

Yeh RF, Gaver VE, Patterson KB, Rezk NL, Baxter-Meheux F, Blake MJ, Eron JJ, Jr, Klein CE, Rublein JC, and Kashuba AD (2006) Lopinavir/ritonavir induces the hepatic activity of cytochrome P450 enzymes CYP2C9, CYP2C19, and CYP1A2 but inhibits the hepatic and intestinal activity of CYP3A as measured by a phenotyping drug cocktail in healthy volunteers. $J$ Acquir Immune Defic Syndr 42:52-60.
Zhou J, Tracy TS, and Remmel RP (2011) Correlation between bilirubin glucuronidation and estradiol-3-gluronidation in the presence of model UDP-glucuronosyltransferase 1A1 substrates/inhibitors. Drug Metab Dispos 39:322-329.

Address correspondence to: Mohamad Shebley, Clinical Pharmacology and Pharmacometrics, AbbVie Inc., 1 N. Waukegan Road, AP31-3, North Chicago, IL 60064. E-mail: mohamad.shebley@abbvie.com 